\title{
Cryo-electron microscopy research at the institute of membrane proteins: current status and future prospects
}

\author{
Ji Won Kim and Jie-Oh Lee* \\ Department of Life Sciences and Institute of Membrane Proteins, POSTECH, Pohang 37673, Republic of Korea \\ ${ }^{*}$ Correspondence: jieoh@postech.ac.kr
}

Transmembrane proteins are among the most important targets for drug discovery. Cryo-EM is particularly useful in membrane protein research because crystallization of target proteins is not necessary. The Institute of Membrane Proteins is currently being constructed at POSTECH to utilize this technological development and facilitate membrane protein research in Korea. Several instruments, including two cryo-EMs will be installed at the institute for public use. Facilities necessary for antibody development against transmembrane proteins will also be installed. Experts, who can operate these instruments as well as help other external scientists, have been hired. In addition to supporting external research, the institute will carry out internal research. Fourteen research groups from universities and national laboratories have currently participated in this project. By collaborating with staff scientists at the institute, these research groups will conduct structural analysis of membrane proteins and develop antibody drugs against them.

\section{INTRODUCTION}

Transmembrane proteins are arguably the most important targets for drug discovery. Approximately $30 \%$ of the human genome encodes transmembrane proteins. This class of proteins includes receptors, transporters, ion channels, and enzymes, which are among the most popular targets that can be used by small molecules and antibody drugs. Transmembrane proteins are of apparent medical importance; however, highresolution structural studies on such proteins are hindered due to many technical obstacles. Firstly, large quantities of highly purified proteins are required for screening crystallization conditions. Production of proteins in quantities sufficient for the crystallization screening process is challenging for most transmembrane proteins. Second, crystallization of these proteins poses additional obstacles. Well-ordered crystals are a prerequisite for high-resolution x-ray crystallographic studies. Transmembrane alpha helices constitute a significant part of $G$ protein-coupled receptors (GPCRs) and other transmembrane proteins. These parts of transmembrane proteins cannot be used for ordered crystallization because they are covered with highly flexible detergent molecules. This surface property of the transmembrane proteins dramatically reduces the chance of obtaining crystals suitable for high-resolution studies.

Recent advancement in technology related to cryo-electron microscopy (EM) allows researchers to overcome a few technical challenges associated with high-resolution structural studies of transmembrane proteins. First, only a few tens of micrograms of proteins are often enough for successful EM sample preparation. This is in sharp contrast to $\mathrm{x}$-ray crystallography, which usually requires 100 - to 1000 -fold more proteins.
Furthermore, crystallization of a target protein is not necessary for cryo-EM, and frozen solutions of the proteins can be directly analyzed. Owing to these advantages, cryo-EM has rapidly become a standard tool for high-resolution structural studies of transmembrane proteins, replacing x-ray crystallography. It has been predicted that structures determined by cryo-EM will surpass those determined by x-ray crystallography in 2024 . Regardless of these apparent advantages, cryo-EM has several disadvantages compared to x-ray crystallography. First, the size of the target proteins should be larger than $\sim 50 \mathrm{kDa}$. Additionally, proteins should be larger than $\sim 100 \mathrm{kDa}$ for routine structural analysis. This is a significant shortcoming of cryo-EM because the most exciting drug discovery targets, such as GPCRs and kinases, are smaller than the current size limits of proteins that can be used in this method. Second, the price of the instrument is very high; hence, only a limited amount of beam time is available for wealthy institutions. This is a significant challenge and appears to be a major obstacle in advancing this relatively new technique.

Structural biology is a crucial field in biological research in Korea. The Pohang Accelerator Laboratory was constructed more than thirty years ago. The first $x$-ray beamline for protein research was launched in 2001 and supported structural biology research in Korea for two decades. Many high caliber reports have been published since then. Regardless of this outstanding progress in synchrotron-based structural biology research, government investment in cryo-EM has been delayed and hindered research activities in Korea. The first cryo-EM machine was installed a couple of years ago. However, this single machine alone could not effectively support all research needs, 
and structural biology studies in Korea had declined in the last several years. To address this problem, a research proposal to establish a research center to study membrane proteins was approved last year. Based on this proposal, the construction of a new institute named "Institute of Membrane Proteins" (IMP) was initiated early this year. The new institute is expected to be operational early next year, in 2021. Two microscopes, TalosGlacios (200 kV) and Titan-Krios (300 kV), will be installed at the IMP shortly after its construction. In addition to these instruments, modern equipment necessary for high-resolution studies on transmembrane proteins and antibody development against them will be installed and operated from early next year. These facilities will be open to public use and will play a critical role in Korea's future structural biology-related research.

\section{SINGLE PARTICLE ANALYSIS OF TRANSMEMBRANE PROTEINS}

Cryo-EM will be the most critical instrument to be operated at the IMP. Traditionally, protein structures have been studied using $\mathrm{x}$-ray crystallography. In x-ray crystallography, crystalline protein samples are irradiated using an intense $x$-ray beam generated in synchrotron sources. Beams diffracted by the crystals are detected as hundreds of thousands of spots using a detector. By measuring the intensities of these spots, the images of proteins from the crystal structures can be reconstituted. Therefore, exploring the required conditions for crystallization is the first step and a prerequisite for high-resolution x-ray crystallographic analysis. Screening of hundreds of thousands of different buffer conditions is necessary to attain conditions that can generate diffraction-quality crystals. For this process, large quantities of highly purified proteins are essential. Unfortunately, the production of protein samples and exploring crystallization conditions are technically challenging for many important target proteins, including transmembrane proteins. Therefore, highresolution structural studies have been nearly impossible for a majority of transmembrane proteins, until a breakthrough related to high-resolution analysis by cryo-EM was uncovered.

Traditionally cryo-EM has been used for low-resolution structural studies of biological samples for several decades. However, due to the advancement of the electron microscopic instrument, the resolution of cryo-EM has dramatically improved, and a resolution greater than 3 -angstrom has become routinely achievable. Because crystallization of a sample is not necessary for cryo-EM analysis, this new technique made structural studies of many transmembrane proteins possible. Typical single particle analysis using cryo-EM begins with the screening of conditions that can produce a thin ice layer having a thickness of a few hundred nanometers. Protein particles are trapped within this layer. The particles are required to be evenly distributed with a random orientation to obtain high-resolution reconstruction of protein structures. It often requires screening of several tens of conditions to find the optimal grid conditions. Recently, commercial instruments utilizing inkjet printing (Jain et al., 2012) or dip pen technology (Ravelli et al., 2020) have been developed. These instruments are expected to reduce the amount of protein necessary for sample screening further and save valuable microscopy time required to screen sample conditions. Dramatic improvement in the resolution of cryo-EM analysis is largely due to direct electron detector technology. Using this method, the images can be split into multiple frames, which allows computer software to preserve high-resolution information of the images by correcting for stage drift and beam-induced motion during image processing (Campbell et al., 2012). It also allows for the correction of radiation damage by down-weighting information in a resolution-dependent manner for each frame. New computer programs are being actively developed that can push the resolution limit even further, i.e., to atomic resolution. This resolution is achieved by correcting loss of focus for each particle in a micrograph, performing Ewald sphere correction, and correcting beam tilt (Glaeser et al., 2011; Grigorieff, 2016; Grant et al., 2018; Russo and Henderson, 2018; Zivanov et al., 2018).

Single-particle analysis of GPCRs has drawn much attention because these transmembrane proteins are arguably the most important targets for drug discovery. GPCRs play crucial roles in regulating cellular physiology and homeostasis. They can act as targets for drug design to be used as therapeutics against various diseases, including cancer, inflammatory, immunological, cardiovascular, and liver diseases, as well as infectious and metabolic diseases. Currently, over 300 candidates are under clinical trials. Sixty of them are targeted against orphan GPCR receptors. Nevertheless, GPCR drugs used in the clinic have targeted only $27 \%$ of non-olfactory GPCRs; therefore, we are expecting more efficient drugs that will target the rest of this protein family.

GPCRs alone are too small in size for cryo-EM analysis. However, those bound to a $G$ protein complex, a universal antibody, $\beta$-arrestin and/or other interacting proteins are larger than the current size limits. Consequently, many structures of the activated forms of GPCRs are determined by single particle analysis employing cryo-EM. In year 2020 alone, the complex structures of $\mathrm{GABA}_{\mathrm{B}}$ receptor (Wu et al., 2014; Koehl et al., 2019; Papasergi-Scott et al., 2020; Shaye et al., 2020), Dopamine D2 receptor (Yin et al., 2020), M2 muscarinic receptor (Maeda et al., 2019; Staus et al., 2020), Neurotenisin receptor (Huang et al., 2020), GLP-1 receptor (Zhao et al., 2020), CCR6 (Wasilko et al., 2020), formylpeptide receptor (Zhuang et al., 2020), PAC1 receptor (Wang et al., 2020), glucagon receptor (Qiao et al., 2020), corticotropin-releasing factor receptor (Ma et al., 2020) and cannabinoid receptor (Hua et al., 2020) were reported (Figure 1). These structures provide valuable information on how these receptors are activated and how they initiate downstream signaling cascades. Furthermore, these protein structures along with their agonists provide a reliable platform to design future drugs against such proteins. Although single particle analysis by cryo-EM is a standard method for analyzing activated GPCRs, 
(a)

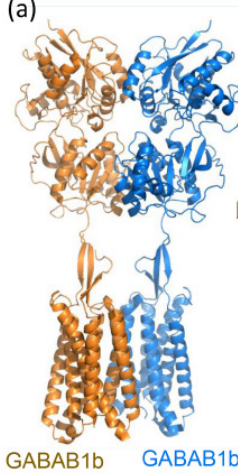

(b)

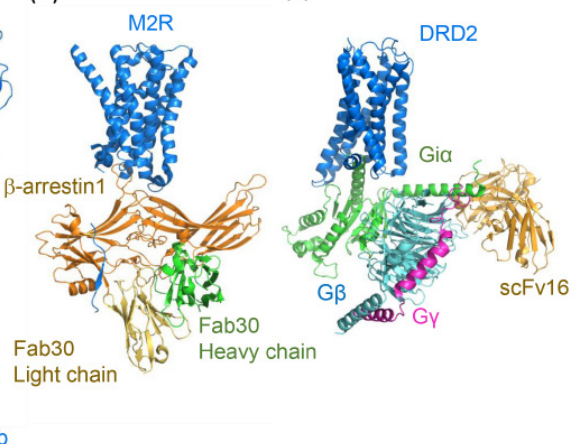

(d)

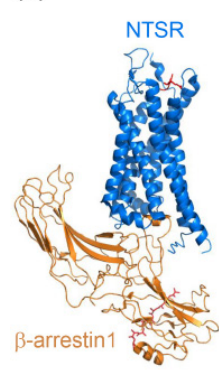

(e)

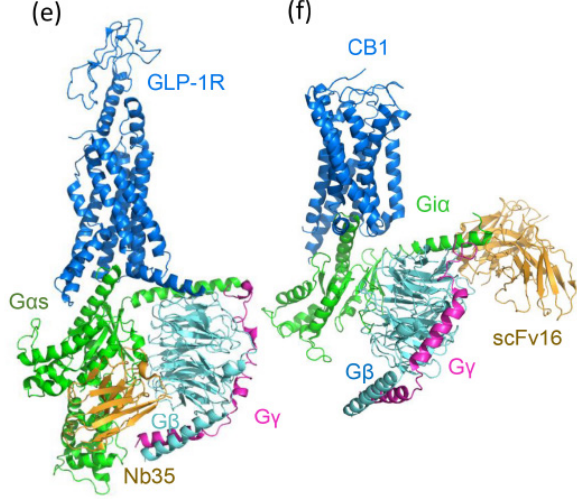

FIGURE 1 I Cryo-EM structures of GPCR complexes. (A) 6W2Y (GABAB1b). (B) 6U1N (M2 Muscarinic receptor-beta arrestin). (C) 6VMS (D2-Dopamin receptor-G-protein complex). (D) 6UP7 (Neurotensin receptor). (E) 6X1A (GLP-1 receptor-G-protein complex). (F) 6KPG (Cannabinoid receptor CB1-G-protein complex).

those bound to antagonists and in the absence of bound $G$ protein analogs are still beyond the size limit of routine single particle analysis. Furthermore, to be used in structure-based drug discovery, the method should be automated and routinely available to the drug design community. Future development of new technology in sample preparation, data collection, and processing is required in this direction. These technologies will be beneficial not only for drug discovery against GPCRs, but also against other important drug targets, such as kinases, E3 ligases, and ion channels.

The advancement of cryo-EM in structural biology has initiated a significant impact on structure-based drug discovery (GarciaNafria and Tate, 2020). A large number of structures of GPCRs, ion channels, and transporters are being solved by cryo-EM. These structures are available for rational design of drugs against diverse diseases. The role of cryo-EM in drug discovery will become more important in the future because new modeling tools based on artificial intelligence will make computational drug design more accurate and useful in drug discovery (Fleming, 2018). Experimental structural information is crucial for precise docking of drug candidates and predicting their affinities for target proteins by computational methods.

TABLE 1 | Cryo-EM and computational facility

\begin{tabular}{|c|c|}
\hline Name & Specification \\
\hline Talos-Glacios & $\begin{array}{l}200 \mathrm{kV} \text { electron gun } \\
\text { Falcon IV direct electron detector }\end{array}$ \\
\hline Titan-Krios & $\begin{array}{l}300 \mathrm{kV} \text { electron gun } \\
\text { K3 Summit direct electron detector }\end{array}$ \\
\hline Chameleon & automatic sample preparation \\
\hline GPU server & $\begin{array}{l}56 \text { RTX2080ti graphic cards } \\
224 \text { CPU cores }\end{array}$ \\
\hline CPU server & $\begin{array}{l}192 \text { CPU cores } \\
\text { Schrodinger Glide software, AutoDock } 3.0\end{array}$ \\
\hline Storage server & 1,008 Tb hard disk array \\
\hline Workstation & 6 workstation, each with 4 RTX2080ti graphic cards \\
\hline
\end{tabular}

\section{CRYO-EM FACILITY AT THE IMP}

Cryo-EM instruments are costly and require experts for their operation. Therefore, small venture companies or universities cannot purchase or use these instruments. To overcome this challenge, publicly available cryo-EM instruments will be installed and operated at the IMP. These microscopes will be available to scientists in universities and industries globally. Two microscopes will be installed at the IMP within a year. A TalosGlacios microscope equipped with a $200 \mathrm{kV}$ electron gun with a Falcon IV direct electron detector has already been installed in POSTECH and will be used mainly for screening purposes (Table 1). For a successful analysis of the high-resolution structures, the protein sample should be trapped in thin ice layers. Obtaining conditions for this, extensive screening of experimental conditions is necessary with the Talos microscopes. The TitanKrios microscope having a $300 \mathrm{kV}$ electron gun and a K3 summit electron detector will be installed for the higher resolution study. Samples optimized using the Talos screening microscope will be analyzed using this microscope, and thousands of images will be obtained for high-resolution image analysis.

A high-performance computation facility equipped with modern graphic processing unit (GPU) chips is essential for cryoEM analysis to process millions of particle images. High-speed GPU-based servers are crucial for this type of image analysis. At the IMP, we will have a rackmount GPU server that can calculate microscopic image reconstitution. Protein structures obtained using this procedure will later be used for drug design. For this kind of analysis, a library of tens of millions of compounds needs to be virtually screened (Forli et al., 2016; Sterling and Irwin, 2015). A CPU with $200 \mathrm{CPU}$ cores will be installed at the IMP, which will assist drug design research conducted by university and industry scientists. Several experts in cryo-EM and computational calculations have been hired and they are currently optimizing the operational protocols.

Sharing cryo-EM equipment in academic and drug design communities is a widely accepted idea. Many synchrotrons, including Diamond, ESRF, SLAC, and others, accept external user applications for cryo-EM analysis. Centers specialized 
in cryo-EM, including NCCAT, S2C2, PNCC, NeCEN, EMBL Heidelberg, have been established and operational. For the pharmaceutical industry, Harwell industrial eBIC and Cambridge Pharma Consortium have five Titan-Krios microscopes, respectively. Some of these instruments are open exclusively to the pharmaceutical industry. UCSF, USC, Tokyo University, Osaka University, Tsinghua University, and others have similar cryo-EM centers, and some of their microscopes are allocated to industrial scientists.

\section{ANTIBODY FACILITY AT THE IMP}

Antibodies are crucial in cryo-EM analysis of proteins due to several reasons. First, the antibodies contribute the cryoEM analysis by increasing the effective size of a target by $\sim 25-50 \mathrm{kDa}$. Although the size of an antibody is much larger than that, in most cases, only the Fv domains of an antibody maintain a rigid structure against the rest of the protein complex when bound to the target protein and, is visible in the density maps. An increase in the size of the target proteins helps find particles in noisy images and orients the particles for image reconstitution. Second, antibodies are also useful in selecting a particular conformation of target proteins that possess multiple conformations. Many proteins can have several conformations for activity and regulation. Therefore, owing to the conformational heterogeneity, particles need to be classified, and only a small fraction of the particles can be used for reconstruction of the protein structure. This increases the number of images required for high-resolution structural studies of target proteins. In worse cases, heterogeneity can considerably reduce the effective resolution of the structures. However, antibodies can be used to selectively bind to one of the available conformations of the proteins and stabilize its structure. This increases the resolution of the structure and reduces analysis time.

Antibodies are important not only for structural analysis but also for drug discovery. Together with small molecule compounds, they are the most important class of drugs available. Since 2001, more than 90 antibodies have been approved for clinical use. Most of these antibodies are targeted to cell surface receptors or ligands bound to them. The generation of clinical quality antibodies is complex and requires collaborative work by experts. This process is particularly tricky in the case of transmembrane proteins, including GPCRs, ion channels, or transporters, for several reasons. First, the production a large number of high-quality antigens for antibody generation is not easy for this class of proteins. Second, only a small part of the protein is available to be used as epitopes. Therapeutic antibodies should be targeted to the exposed parts of these proteins. Extracellular domains of GPCRs, ion channels, or transporters often constitute only a small part of the protein surface; hence, the chances of obtaining high-affinity antibodies are significantly smaller. Third, many transmembrane proteins are essential for cellular functions, and their amino acid sequences are often evolutionarily conserved. Consequently, a high sequence homology of human proteins with mouse proteins reduces the possibility of obtaining monoclonal antibodies of high affinity and therapeutic activity.

At the IMP, we will install research equipment essential for antibody generation. These include a large-scale cell culture facility, many HPLCs, gene cloning instruments, and equipment required to perform binding and activity assays. We have hired several experts having many years of antibody-related research experience to operate these sophisticated instruments. We will also collaborate with other laboratories for this purpose,

(a)

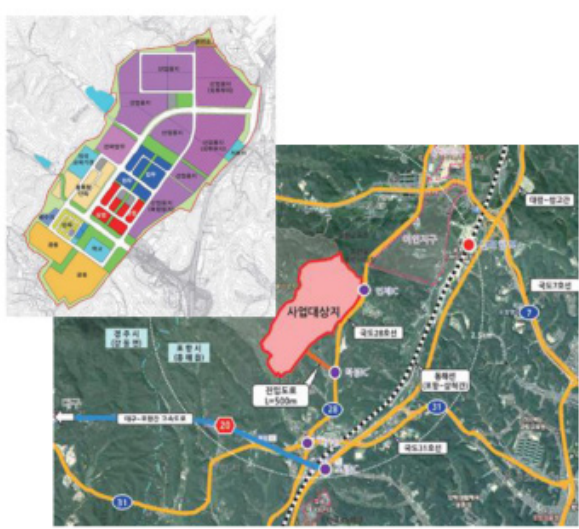

(b)

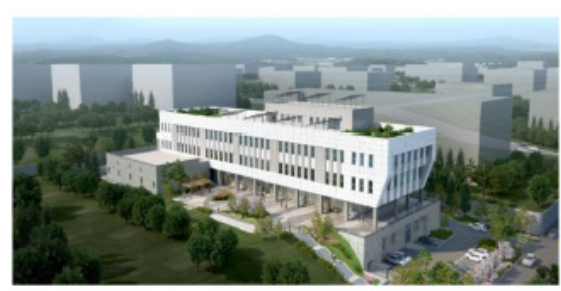

(c)

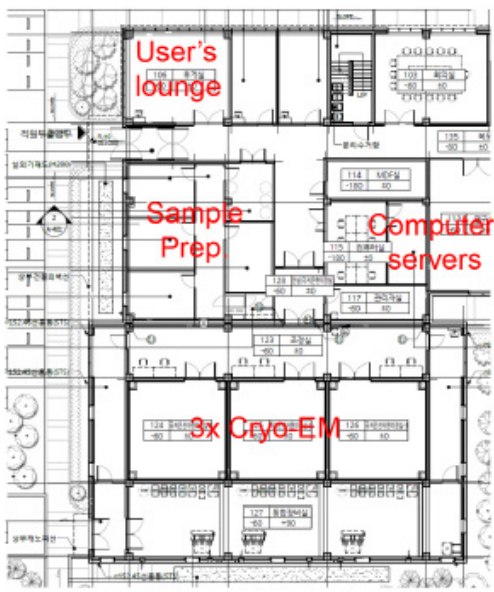

FIGURE 2 I Construction plan of IMP. (A) Location of IMP. It is located in the Pohang free economic zone close to the KTX station and Pohang Accelerator Laboratory. (B) Bird eye view of the IMP building. (C) Floor plan of the cryoEM facility. IMP has three rooms for cryo-EM and separate rooms for sample preparation and computational facility. 
including the mouse facility at POSTECH and plant vaccine laboratory located within a short distance. The antibody facility at the IMP will support not only internal research, but also external scientists, who have limited equipment and experience.

\section{CONSTRUCTION SCHEDULE AND FLOOR PLAN}

Construction of the IMP building was initiated early this year and will be completed by January 2021. The building has four stories (Figure 2). Environment control and electrical shops will be located in the basement. The cryo-EMs will be installed on the first floor. The computational facility is located near the cryo-EM to support image analysis and virtual screening. All the antibody and protein facilities will be located on the second floor. The third floor is reserved for startup companies, and a small dormitory for accommodating outside researchers is located on one side of the floor.

\section{CONCLUSION}

Structural biology-based research activities on cryo-EM have been recently initiated in Korea. There is a long way to go before Korea gains a competitive edge in this rapidly growing field. To support this mission, the IMP will include the essential instruments installed and experts to operate them. This equipment will mainly support structural studies related to important drug targets, such as GPCRs, ion channels, and transporters. The institute will be located within a short distance from the Pohang Accelerate Laboratory and the immunology facility of POSTECH. A close collaboration with these institutions will be pivotal to achieve the ambitious mission toward efficient structural biology-based research in Korea.

\section{ACKNOWLEDGEMENTS}

This research was supported by the National Research Foundtion of Korea (NRF 2019M3E5D6066058 and NRF2017 M3A9F6029753).

\section{CONFLICT OF INTEREST}

The authors declare no conflicts of interest.

Original Submission: Dec 1, 2020

Revised Version Received: Dec 16, 2020

Accepted: Dec 17, 2020

\section{REFERENCES}

Campbell, M.G., Cheng, A., Brilot, A.F., Moeller, A., Lyumkis, D., Veesler, D., Pan, J., Harrison, S.C., Potter, C.S., Carragher, B., and Grigorieff, N. (2012). Movies of ice-embedded particles enhance resolution in electron cryo-microscopy. Structure 20, 1823-1828.

Fleming, N. (2018). How artificial intelligence is changing drug discovery. Nature 557, S55-S57.

Forli, S., Huey, R., Pique, M.E., Sanner, M.F., Goodsell, D.S., and Olson, A.J. (2016). Computational protein-ligand docking and virtual drug screening with the AutoDock suite. Nat Protoc 11, 905-919.

García-Nafría, J., and Tate, C.G. (2020). Cryo-electron microscopy: moving beyond $\mathrm{X}$-ray crystal structures for drug receptors and drug development. Annu Rev Pharmacol Toxicol 60, 51-71.

Glaeser, R.M., Typke, D., Tiemeijer, P.C., Pulokas, J., and Cheng, A. (2011). Precise beam-tilt alignment and collimation are required to minimize the phase error associated with coma in high-resolution cryoEM. J Struct Biol 174, 1-10.

Grant, T., Rohou, A., and Grigorieff, N. (2018). cisTEM, user-friendly software for single-particle image processing. Elife 7, e35383.

Grigorieff, N. (2016). Frealign: an exploratory tool for single-particle CryoEM. Methods Enzymol 579, 191-226.

Hua, T., Li, X., Wu, L., Iliopoulos-Tsoutsouvas, C., Wang, Y., Wu, M., Shen, L., Johnston, C.A., Nikas, S.P., Song, F., Song, X., Yuan, S., Sun, Q., Wu, Y., Jiang, S., et al. (2020). Activation and signaling mechanism revealed by cannabinoid receptor-Gi complex structures. Cell 180, 655665.e618.

Huang, W., Masureel, M., Qu, Q., Janetzko, J., Inoue, A., Kato, H.E., Robertson, M.J., Nguyen, K.C., Glenn, J.S., Skiniotis, G., and Kobilka, B.K. (2020). Structure of the neurotensin receptor 1 in complex with $\beta$-arrestin 1. Nature 579, 303-308.

Jain, T., Sheehan, P., Crum, J., Carragher, B., and Potter, C.S. (2012). Spotiton: a prototype for an integrated inkjet dispense and vitrification system for cryo-TEM. J Struct Biol 179, 68-75.

Koehl, A., Hu, H., Feng, D., Sun, B., Zhang, Y., Robertson, M.J., Chu, M., Kobilka, T.S., Laeremans, T., Steyaert, J., Tarrasch, J., Dutta, S., Fonseca, R., Weis, W.I., Mathiesen, J.M., et al. (2019). Structural insights into the activation of metabotropic glutamate receptors. Nature 566, 79-84.

Ma, S., Shen, Q., Zhao, L.H., Mao, C., Zhou, X.E., Shen, D.D., de Waal, P.W., Bi, P., Li, C., Jiang, Y., Wang, M.W., Sexton, P.M., Wootten, D., Melcher, K., Zhang, Y., et al. (2020). Molecular basis for hormone recognition and activation of corticotropin-releasing factor receptors. $\mathrm{Mol}$ Cell 77, 669-680.e664.

Maeda, S., Qu, Q., Robertson, M.J., Skiniotis, G., and Kobilka, B.K. (2019). Structures of the M1 and M2 muscarinic acetylcholine receptor/G-protein complexes. Science 364, 552-557.

Papasergi-Scott, M.M., Robertson, M.J., Seven, A.B., Panova, O., Mathiesen, J.M., and Skiniotis, G. (2020). Structures of metabotropic GABAB receptor. Nature $\mathbf{5 8 4}, \mathbf{3 1 0 - 3 1 4 .}$

Qiao, A., Han, S., Li, X., Li, Z., Zhao, P., Dai, A., Chang, R., Tai, L., Tan, Q., Chu, X., Ma, L., Thorsen, T.S., Reedtz-Runge, S., Yang, D., Wang, M.W., et al. (2020). Structural basis of Gs and Gi recognition by the human glucagon receptor. Science 367, 1346-1352.

Ravelli, R.B.G., Nijpels, F.J.T., Henderikx, R.J.M., Weissenberger, G., Thewessem, S., Gijsbers, A., Beulen, B., López-Iglesias, C., and Peters, P.J. (2020). Cryo-EM structures from sub-nl volumes using pin-printing and jet vitrification. Nat Commun 11, 2563.

Russo, C.J., and Henderson, R. (2018). Ewald sphere correction using a single side-band image processing algorithm. Ultramicroscopy 187, 2633.

Shaye, H., Ishchenko, A., Lam, J.H., Han, G.W., Xue, L., Rondard, P., Pin, J.P., Katritch, V., Gati, C., and Cherezov, V. (2020). Structural basis of the activation of a metabotropic GABA receptor. Nature 584, 298-303.

Staus, D.P., Hu, H., Robertson, M.J., Kleinhenz, A.L.W., Wingler, L.M., Capel, W.D., Latorraca, N.R., Lefkowitz, R.J., and Skiniotis, G. (2020). Structure of the M2 muscarinic receptor- $\beta$-arrestin complex in a lipid nanodisc. Nature 579, 297-302.

Sterling, T., and Irwin, J.J. (2015). ZINC 15--ligand discovery for everyone. J Chem Inf Model 55, 2324-2337. 
Wang, J., Song, X., Zhang, D., Chen, X., Li, X., Sun, Y., Li, C., Song, Y., Ding, Y., Ren, R., Harrington, E.H., Hu, L.A., Zhong, W., Xu, C., Huang, X., et al. (2020). Cryo-EM structures of PAC1 receptor reveal ligand binding mechanism. Cell Res 30, 436-445.

Wasilko, D.J., Johnson, Z.L., Ammirati, M., Che, Y., Griffor, M.C., Han, S., and $\mathrm{Wu}, \mathrm{H}$. (2020). Structural basis for chemokine receptor CCR6 activation by the endogenous protein ligand CCL20. Nat Commun 11, 3031.

Wu, H., Wang, C., Gregory, K.J., Han, G.W., Cho, H.P., Xia, Y., Niswender, C.M., Katritch, V., Meiler, J., Cherezov, V., Conn, P.J., and Stevens, R.C. (2014). Structure of a class C GPCR metabotropic glutamate receptor 1 bound to an allosteric modulator. Science 344, 58-64.

Yin, J., Chen, K.M., Clark, M.J., Hijazi, M., Kumari, P., Bai, X.C., Sunahara, R.K., Barth, P., and Rosenbaum, D.M. (2020). Structure of a D2 dopamine
receptor-G-protein complex in a lipid membrane. Nature 584, 125-129.

Zhao, P., Liang, Y.L., Belousoff, M.J., Deganutti, G., Fletcher, M.M., Willard, F.S., Bell, M.G., Christe, M.E., Sloop, K.W., Inoue, A., Truong, T.T., Clydesdale, L., Furness, S.G.B., Christopoulos, A., Wang, M.W., et al. (2020). Activation of the GLP-1 receptor by a non-peptidic agonist. Nature 577, 432-436.

Zhuang, Y., Liu, H., Edward Zhou, X., Kumar Verma, R., de Waal, P.W., Jang, W., Xu, T.H., Wang, L., Meng, X., Zhao, G., Kang, Y., Melcher, K., Fan, H., Lambert, N.A., Eric Xu, H., et al. (2020). Structure of formylpeptide receptor 2-Gi complex reveals insights into ligand recognition and signaling. Nat Commun 11, 885.

Zivanov, J., Nakane, T., Forsberg, B.O., Kimanius, D., Hagen, W.J., Lindahl, E., and Scheres, S.H. (2018). New tools for automated highresolution cryo-EM structure determination in RELION-3. Elife 7, e42166. 